Methodik und Ergebnisse eines Nachhaltigkeits-Ratings von Staaten

\section{Deutschland ist nur Mittelmaß}

\section{Festverzinsliche Wertpapiere sind ein geeignetes Mittel, um die Risiken der Ak- tienanlage auszugleichen. Institutionellen Anlegern, die nachhaltig investieren wollen, steht nun ein neves Instrument im festverzinslichen Anlagenbereich zur Verfügung: Das von oekom research entwickelte ethisch-ökologische Country Rating.}

Von Matthias Bönning m Rahmen einer halbjährigen Studie wurden für das Country Rating die 30 Staaten der Organisation für wirtschaftliche Entwicklung und Zusammenarbeit (OECD) und Russland von der oekom research AG untersucht. In Zusammenarbeit mit ExpertInnen aus Wissenschaft und Forschung wurden für das Rating 130 Indikatoren ausgewählt, um die ökologische und soziale Situation eines Landes realistisch abzubilden. Die Bewertung der Indikatoren erfolgte auf Basis quantitativer und qualitativer Daten von Organisationen wie der OECD, den Vereinten Nationen, der Weltgesundheitsorganisation oder der Menschenrechtsinitiative Amnesty International. Multipliziert mit dem jeweiligen Gewicht der Indikatoren ergibt sich eine Gesamtnote, die sich jeweils zur Hälfte aus dem Umwelt- und dem Sozialbereich speist. Während sich die Bewertung der qualitativen Kriterien auf der Grundlage der jeweiligen Performance eines Staates im Vergleich zu anderen Staaten ergibt, wird bei quantitativen Indikatoren (zum Beispiel Ressourcenverbräuche) zusätzlich der Trend im Zeitablauf innerhalb eines Landes benotet.

Das Nachhaltigkeits-Rating versteht sich als Ergänzung zu klassischen Credit Ratings. Insofern wurde bei der Bewertung auf finanzwirtschaftliche Indikatoren bewusst verzichtet. Der Umweltbereich des Ratings orientiert sich von der Struktur her am Pressure-State-Response-Konzept der OECD. Analysiert werden neben den Umweltbelastungen - also beispielsweise Boden- und Wasserverschmutzung, Luftemissionen und Energiemix - der Umweltzustand im jeweiligen Land sowie die Reaktion von Politik und Gesellschaft. Diese fließt über den Grad der Institutionalisierung von Umweltschutz, über öffentliche Umweltprogramme sowie über die Teilnahme an internationalen Abkommen in die Bewertung ein.

Die konzeptionelle Basis für den Sozialbereich liefern die „Seven Freedoms“ des United Nations
Development Programme (UNDP). Ausgehend von den politischen und gesellschaftlichen Rahmenbedingungen werden die tatsächlichen sozialen Bedingungen sowie die Infrastruktur des Landes betrachtet. Ersteres umfasst grundlegende Strukturen wie das politische System sowie die Gewährung und Einhaltung von Menschenrechten. Dazu werden die öffentlichen Ausgaben, die Existenz nicht-staatlicher Institutionen wie Gewerkschaften und Medien sowie Korruption und Geldwäsche untersucht. Im Rahmen der sozialen Bedingungen in einem Land bewertet das Rating Bereiche wie Gleichberechtigung, Gesundheit, Bildung und Arbeitsbedingungen.

\section{Skandinavische Länder führen}

In dem aktuellen Ranking belegen vier skandinavische Staaten die ersten Plätze, Russland bildet das Schlusslicht. Die Analysten von oekom research bescheinigen Spitzenreiter Norwegen eine hervorragende Gesamtperformance, die im sozialen Bereich besonders hervorzuheben sei. Beispielhaft seien die für europäische Verhältnisse geringe Staatsverschuldung und die geringe Arbeitslosigkeit bei generell guten Arbeitsbedingungen.

Deutschland und die USA sind lediglich im Mittelfeld platziert. Der enttäuschende zwölfte Rang Deutschlands ist vor allem auf Defizite im Bereich Bildung (Stichwort PISA-Studie) und auf die hohe Arbeitslosigkeit zurückzuführen. Auch im Umweltbereich bleiben die Deutschen deutlich hinter einer Vielzahl von OECD-Staaten zurück. Dafür verantwortlich ist die geringe Artenvielfalt und der niedrige Anteil geschützter Naturflächen. Vorbildlich ist Deutschland beim effizienten Umgang mit knappen Ressourcen: bezogen auf die Wirtschaftsleistung hat das Land relativ geringe Schadstoffemissionen sowie niedrige Abfallaufkommen und Energieverbräuche.

Die USA liegen im Gesamtranking noch dahinter auf Platz 17. Sie zeigen insbesondere bei den The- men Menschenrechte und Entwicklungshilfe deutliche Schwachstellen: Während die USA als eines der reichsten Länder der Erde für letztere in den vergangenen Jahren nur 0,1 Prozent ihres Bruttoinlandsproduktes ausgaben, war es in Norwegen der neunfache Satz. In einigen anderen sozialen Bereichen sind die USA aus Sicht der Analysten jedoch beispielhaft, so etwa bei der Pressefreiheit. Hinsichtlich der Kohlendioxidemissionen schneiden die USA besonders schlecht ab: Bezogen auf jede Einheit erzeugter Wirtschaftsleistung emittieren die Amerikaner zweieinhalbmal soviel Kohlendioxid wie die Schweiz - der Spitzenreiter in diesem Feld.

In der Vergangenheit haben bereits verschiedene Institutionen Konzepte entwickelt, um Staaten auf der Basis von Nachhaltigkeitsaspekten vergleichen zu können. Jedoch beschränken sich Ansätze wie der Human Development Index des UNDP oder der Environmental Sustainability Index des World Economic Forum nur auf spezielle Teilbereiche der nachhaltigen Entwicklung.

Ein in Bezug auf die Zielgruppe Investoren mit dem Country Rating von oekom research vergleichbares Produkt hat dagegen vor kurzem die Züricher Kantonalbank veröffentlicht: Mit 50 ausschließlich quantitativen Einzelindikatoren, die speziell im Hinblick auf die Bedingungen in OECD-Ländern ausgewählt wurden, wird versucht, die wesentlichen, außerökonomischen Bereiche der Nachhaltigkeit abzudecken. Im Gegensatz dazu wurde das Country Rating von oekom research bewusst offen auch für Nicht-Industriestaaten konzipiert, um entsprechende Kundenwünsche bedienen zu können. Auch wäre durch eine Beschränkung auf quantitative Indikatoren keine ausreichende Beurteilung von qualitativen Untersuchungsbereichen wie die Einhaltung von Menschenrechten möglich gewesen. Schließlich können Interessenten selbst zusätzliche Ausschlusskriterien vorgeben. Das Country Rating ermöglicht damit institutionellen Anlegern, ihre Staatsanleihen in Portfolios und Fonds nachhaltig auszurichten.

\section{Der Autor}

Matthias Bönning ist Analyst bei oekom research. Kontakt: oekom research AG, Goethestraße 28, 80336 München, Tel. 089/ 544184-54, Fax -99 E-Mail: boenning@oekom-research.com 
(c) 20I0 Authors; licensee IÖW and oekom verlag. This is an article distributed under the terms of the Creative Commons Attribution Non-Commercial No Derivates License (http://creativecommons.org/licenses/by-nc-nd/3.o/), which permits unrestricted use, distribution, and reproduction in any medium, provided the original work is properly cited. 